\title{
Improved Dynamic Characteristics on Four-Wave Mixing Wavelength Conversion in Light-Holding SOAs
}

\author{
Jyh-Tsung Hsieh, Pei-Miin Gong, San-Liang Lee, Member, IEEE, and Jingshown Wu, Senior Member, IEEE
}

\begin{abstract}
We investigate theoretically and experimentally the dynamic characteristics of the wavelength conversion that is based on four-wave mixing (FWM) in a light-holding semiconductor optical amplifier (LHSOA). Two FWM conversion techniques, including the single-pump (SP) and two orthogonal-polarized pumps (OPP) schemes, are investigated using a sophisticated time-domain simulation model. The simulation indicates that the holding light gives an improved eye opening and signal-to-noise ratio for both the SP and OPP schemes. The chirp response for both schemes are also presented. The performance improvement are verified from dynamic measurements such as eye diagram and bit-error-rate (BER) tests. Clearer eye openings and reduced power penalties are obtained with the injection of a holding light. By comparing to the experimental results, we also prove that our numerical modeling can predict the device performance and can be used for optimizing the wavelength conversion process.
\end{abstract}

Index Terms-Four-wave mixing (FWM), holding light, orthogonal-polarized pumps (OPPs), semiconductor optical amplifiers (SOAs), wavelength conversion.

\section{INTRODUCTION}

$\mathbf{T}$ HE use of semiconductor optical amplifiers (SOAs) as wavelength converters has been studied extensively both theoretically and experimentally. The wavelength conversion by virtue of the four-wave mixing (FWM) process in an SOA offers advantages such as multiwavelength conversion capability [1], transparency to the modulation format and data rate, and fiber dispersion compensation and so on. However, this technique typically suffers from poor conversion efficiency, narrow conversion range, and signal degradation caused by the amplified spontaneous emission (ASE) noise in the devices.

Significant improvement on the signal-to-background-noise ratio (SBR) and the conversion wavelength range is required for practical applications. High conversion efficiency is attainable from an SOA of very high gain [2], and the conversion range can be improved with the dual-pump techniques [3], [4]. Wave-

Manuscript received December 22, 2003; revised June 15, 2004. This work was supported in part by the National Science Council and the Ministry of Education, Taiwan, R.O.C., under Grants NSC91-2215-E-011-001 and 89EFA06-2-4-7.

J.-T. Hsieh and J. Wu are with the Graduate Institute of Communication Engineering and Department of Electrical Engineering, National Taiwan University, Taipei 106-17, Taiwan, R.O.C. (e-mail: jyh_tsung@ @ pchome.com.tw).

P.-M. Gong and S.-L. Lee are with the Department of Electronic Engineering, National Taiwan University of Science and Technologies, Taipei 106-17, Taiwan, R.O.C.

Digital Object Identifier 10.1109/JSTQE.2004.835300 length conversion over $80 \mathrm{~nm}$ with constant conversion efficiency was demonstrated with two orthogonal-polarized pumps (OPP) [3]. Despite this, the conversion efficiency and SBR of a dual-pump scheme are still low without using an ultrahigh-gain SOA.

The FWM conversion efficiency in SOAs is dominant by the ultrafast relaxation-related processes. The conversion efficiency is linearly proportional to the unsaturated gain and the square of the saturation power [5]. In general, increasing the gain and/or saturation power of an SOA will enhance the conversion efficiency. However, the gain and saturation power usually need to be compromised in designing an SOA. Moreover, the maximum gain is usually limited by the ASE and the thermal effect. The typical fiber-to-fiber gain is around $20 \mathrm{~dB}$ for commercial SOAs. Therefore, it is hard to obtain an SOA with ultrahigh gain and saturation power. Instead, a holding light can be utilized to extend the saturation power, and in turn allow obtaining high conversion efficiency in an SOA with moderate gain.

We have demonstrated experimentally [6] and theoretically [7] that the static characteristics, including saturation intensity and FWM wavelength conversion efficiency, of an SOA could be raised with a holding light. The conversion efficiency can be enhanced by larger than $5 \mathrm{~dB}$ when the SOA is biased at transparency for a $23-\mathrm{dBm}$ and $1480-\mathrm{nm}$ assist light. The holding light can also improve the signal-to-background-noise ratio (SBR) by about $3 \mathrm{~dB}$.

In this paper, we focus on the dynamic properties of a light-holding SOA (LHSOA) for both single-pump (SP) and OPP schemes. The dynamic response is analyzed by modifying the static model to enable time-domain simulation [7]. Both theoretical and empirical results prove that a holding light also provides performance improvements for dynamic responses, including the converted waveforms (eye diagrams) and bit-error rates (BERs). The improvements arise mainly from the enhanced conversion efficiency and SBR.

The structure of the paper is as follows. In Section II, the large-signal static and dynamic models based on the FWM coupled equations and rate equations are described in detail. The experimental setup used to examine the dynamic response of the single-pump FWM wavelength conversion in LHSOAs is introduced in Section III. We explain the experimental data such as eye diagram and BER in combination with our simulation results in Section IV. Section V discusses the performance improvement for OPP FWM wavelength conversion using our developed model. Finally, conclusions are given in Section VI. 


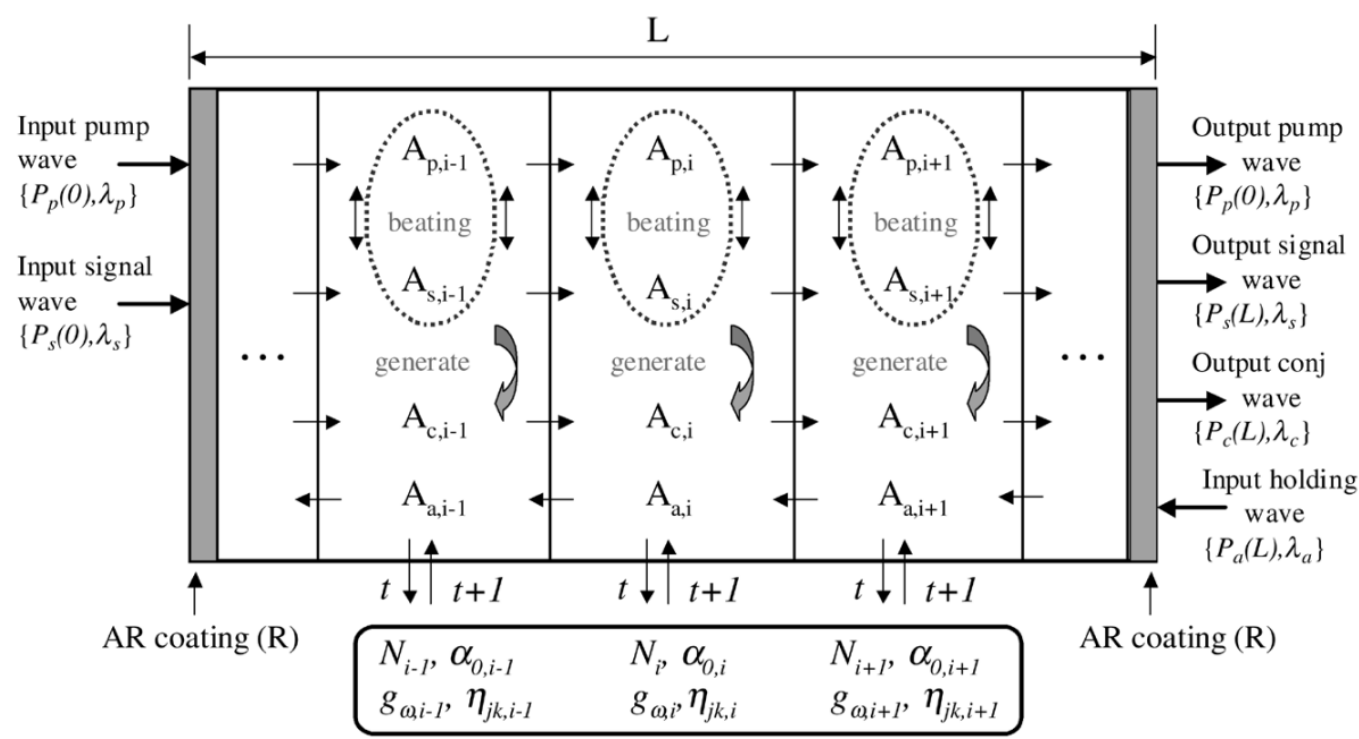

Fig. 1. Schematic diagram of the large-signal dynamic SOA model for single-pump scheme.

\section{MODELING FWM IN SOAS}

\section{A. General Formalism}

Fig. 1 shows a schematic of our time-domain large-signal SOA model, which is extended from the static model [7]. The model is based on position-dependent rate equations for the carrier density and the optical propagation equations in both directions for the whole spectrum of amplified spontaneous emission and injected signals. The SOA is assumed to have negligible reflectivity on the end facets and, therefore, no reflected waves are considered. The SOA is segmented into a number of short sections. The carrier density $N_{i}(t)$ in section $i$ at time $t$ is evaluated by the rate equation

$$
\frac{d N_{i}}{d t}=\frac{J}{e d}-R\left(N_{i}\right)-\sum_{\omega} \Gamma v_{g} g_{m i}\left(\lambda_{\omega}\right) S_{\omega, i}-R_{\mathrm{ASE}}\left(N_{i}\right)
$$

where $J$ is the current density, $e$ is the electron charge, $d$ is the active region thickness, $\Gamma$ is the confinement factor, $g$ is the material gain, $S$ is the average photon density, and the index $\omega$ corresponds to different input lights, including the pumps, signal, conjugate, and holding lights. The average photon density $S_{\omega, i}$ is calculated by

$$
S_{\omega, i}=\frac{\left|A_{\omega, i}\right|^{2}+\left|A_{\omega, i+1}\right|^{2}}{2 v_{g} E_{\omega} A_{\text {cross }}}
$$

where $A_{\omega, i}$ is the traveling wave amplitudes for pump, signal, conjugate, and holding light; $E_{\omega}$ is photon energy; and $A_{\text {cross }}$ is the cross sectional area of the active layer.

The recombination rate depends on the carrier density and is given by

$$
R\left(N_{i}\right)=A N_{i}+B N_{i}^{2}+C N_{i}^{3}
$$

where the coefficients $A, B$, and $C$ stand for the unimolecular recombination, bimolecular radiative recombination, and the Auger recombination process, respectively. In order for modeling the transparent condition, the asymmetric gain spectrum

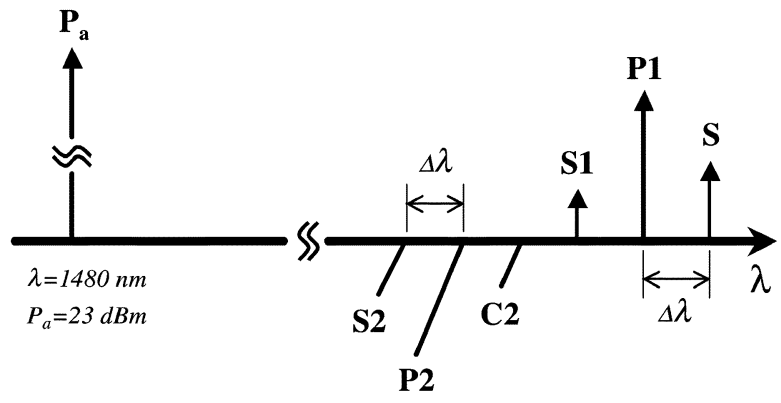

Fig. 2. Schematic diagram of wavelength allocation in the OPP scheme.

is assumed to be cubic and the material gain is approximated by [8]

$$
\begin{aligned}
g_{m \omega, i} & \equiv g_{m}\left(N_{i}, \lambda_{\omega}\right) \\
& =a_{1}\left(N_{i}-N_{0}\right)-a_{2}\left(\lambda_{\omega}-\lambda_{N}\right)^{2}+a_{3}\left(\lambda_{\omega}-\lambda_{N}\right)^{3}
\end{aligned}
$$

where $a_{1}, a_{2}$, and $a_{3}$ are gain constants and $N_{0}$ is the carrier density at transparency for the peak wavelength $\lambda_{N}$, which is assumed to shift linearly with the carrier density, i.e., $\lambda_{N}=$ $\lambda_{0}-a_{4} \cdot\left(N-N_{0}\right)$, with $\lambda_{0}$ being the peak wavelength at transparency. Nonlinear gain compression is included in the model through (1) where the carrier density is influenced by the total photon density of the SOA given by the stimulated emission and the ASE. Note that (4) allows modeling the wavelength-dependent transparent carrier density, which most previous analysis fails to account for. The material loss coefficient $\alpha_{a}\left(\mathrm{~m}^{-1}\right)$ in the active layer is modeled to be carrier-density dependent [7], [9]. The inclusion of the carrier-dependent loss is important for considering the effects of using a holding light. The net modal gain is given by [10]

$$
g_{\omega, i}=g\left(N_{i}, \lambda_{\omega}\right)=\Gamma g_{m \omega, i}-\alpha_{0 i}
$$

where $\alpha_{0}$ includes the loss of the active layers and the cladding layers.

The ASE spectrum is divided into a number of spectral slices in the numerical analysis. The ASE recombination rate $R_{\mathrm{ASE}, i}$ is calculated by summing up the contributions from the forward 
and backward propagating ASE in each section over the whole spectrum [7]. The ASE recombination rate $R_{\mathrm{ASE}, i}$ is given by

where

$$
\begin{aligned}
R_{\mathrm{ASE}, i} & =R_{\mathrm{ASE}}\left(N_{i}\right) \\
& =\sum_{\nu} \Gamma \cdot g_{m}\left(N_{i}, \nu\right) \cdot \frac{P_{\mathrm{ASE}, i}(\nu)}{(h \nu) \cdot A_{\mathrm{cross}}}
\end{aligned}
$$

$$
\begin{aligned}
P_{\mathrm{ASE}, i}(\nu)=G_{i}(\nu) P_{\mathrm{ASE}, i-1}+h \nu \cdot \Delta \nu \cdot n_{\mathrm{sp}} \\
\cdot\left[G_{i}(\nu)-1\right]\left(\frac{\Gamma g_{m}\left(N_{i}, \nu\right)}{\Gamma g_{m}\left(N_{i}, \nu\right)-\alpha_{0 i}}\right)
\end{aligned}
$$

with $n_{\mathrm{sp}}=\bar{N}(z) /\left(\bar{N}(z)-N_{0}\right)$ [11]-[13].

\section{B. Mixing Geometry and Coupled Wave Equations}

We first refer our analysis to the OPP FWM scheme. The configuration of the OPP scheme considered in this paper is shown in Fig. 2. The first pump (P1), at wavelength $\lambda_{p 1}$, is polarized parallel to the signal (S). A second pump (P2), at wavelength $\lambda_{p 2}$ and in a polarization orthogonal to $\mathrm{P} 1$ (and, hence, to $\mathrm{S}$ ), is simultaneously injected into the amplifier. We define the wavelength difference between the $\mathrm{S}$ and $\mathrm{P} 1$ to be $\Delta \lambda$. The conversion efficiency is mainly determined by the value of $\Delta \lambda$.

We consider nondegenerate FWM wavelength conversion in a traveling-wave SOA with collinearly injected pump and signal waves carrying optical powers $P_{p 1}, P_{p 2}$, and $P_{s}$, respectively. The FWM interaction between $\mathrm{P} 1$ and $\mathrm{S}$ generates a signal wave at wavelength $\lambda_{s 1}=2 \lambda_{p 1}-\lambda_{s}$. The FWM signal at $2 \lambda_{s}-\lambda_{p 1}$ is neglected, assuming that the signal power is much smaller than the pump power. Simultaneously, the resultant beating grating will scatter P2 to induce the extra FWM signals S2 and C2 at wavelengths $\lambda_{s 2}=\lambda_{p 2}-\Delta \lambda$ and $\lambda_{c 2}=\lambda_{p 2}+\Delta \lambda$, respectively.

To simplify the analysis, we adopt a phenomenological model to account for the contribution of various nonlinear mechanisms to the FWM process. Following procedures similar to those used in Agrawal's treatment [14], the quasi-steady-state evolution of the pump, signal, and conjugate wave amplitudes $A_{i}, i=$ $s 1, p 1, s, s 2, p 2, c 2$ is given by the coupled equations [15]

$$
\begin{aligned}
\frac{d A_{s 1}}{d z}= & \frac{1}{2}\left[\left\{\Gamma g_{m s_{1}}\left(1-i \alpha_{\mathrm{CM}}\right)-\alpha_{0}\right\}\right. \\
& \left.-\Gamma g_{m s_{1}}\left(\eta_{s 1 p 1}\left|A_{p 1}\right|^{2}+\eta_{s 1 s}\left|A_{s}\right|^{2}\right)\right] A_{s 1} \\
& -\Gamma \frac{g_{m s_{1}}}{2}\left[\eta_{p 1 s} A_{p 1}^{2} A_{s}^{*}+\eta_{s 2 p 2} A_{p 1} A_{s 2} A_{p 2}^{*}\right. \\
& \left.+\eta_{p 2 c 2} A_{p 1} A_{p 2} A_{c 2}^{*}+\eta_{s 2 c 2} A_{s} A_{s 2} A_{c 2}^{*}\right] \\
\frac{d A_{p 1}}{d z}= & \frac{1}{2}\left[\left\{\Gamma g_{m p_{1}}\left(1-i \alpha_{\mathrm{CM}}\right)-\alpha_{0}\right\}\right. \\
& \left.-\Gamma g_{m p_{1}}\left(\eta_{p 1 s 1}\left|A_{s 1}\right|^{2}+\eta_{p 1 s}\left|A_{s}\right|^{2}\right)\right] A_{p 1} \\
& -\Gamma \frac{g_{m p_{1}}}{2}\left[\left(\eta_{s 1 p 1}+\eta_{s p 1}\right) A_{s 1} A_{s} A_{p 1}^{*}\right. \\
& +\eta_{s 2 p 2} A_{s} A_{s 2} A_{p 2}^{*}+\eta_{p 2 s 2} A_{s 1} A_{p 2} A_{s 2}^{*} \\
& \left.+\eta_{p 2 c 2} A_{s} A_{p 2} A_{c 2}^{*}+\eta_{c 2 p 2} A_{s 1} A_{c 2} A_{p 2}^{*}\right] \\
\frac{d A_{s}}{d z}= & \frac{1}{2}\left[\left\{\Gamma g_{m s}\left(1-i \alpha_{\mathrm{CM}}\right)-\alpha_{0}\right\}\right. \\
& \left.-\Gamma g_{m s}\left(\eta_{s s 1}\left|A_{s 1}\right|^{2}+\eta_{s p 1}\left|A_{p 1}\right|^{2}\right)\right] A_{s} \\
& -\Gamma \frac{g_{m s}}{2}\left[\eta_{p 1 s 1} A_{p 1}^{2} A_{s 1}^{*}+\eta_{p 2 s 2} A_{p 1} A_{p 2} A_{s 2}^{*}\right. \\
& \left.+\eta_{c 2 p 2} A_{p 1} A_{c 2} A_{p 2}^{*}+\eta_{c 2 s 2} A_{s 1} A_{c 2} A_{s 2}^{*}\right]
\end{aligned}
$$

TABLE I

Device AND Material Parameters

\begin{tabular}{cccc}
\hline Symbol & Description & Value & Unit \\
\hline$L$ & SOA length & $1000 \times 10^{-6}$ & $\mathrm{~m}$ \\
$w$ & Active layer width & $1.5 \times 10^{-6}$ & $\mathrm{~m}$ \\
$d$ & Active layer thickness & $0.18 \times 10^{-6}$ & $\mathrm{~m}$ \\
$A$ & Unimolecular recombination constant & $1 \times 10^{8}$ & $\mathrm{~s}^{-1}$ \\
$B$ & Bimolecular recombination constant & $2.5 \times 10^{-17}$ & $\mathrm{~m}^{3} / \mathrm{s}$ \\
$\mathrm{C}$ & Auger recombination constant & $9.4 \times 10^{-41}$ & $\mathrm{~m}^{6} / \mathrm{s}$ \\
$a_{1}$ & Material gain constant & $2.5 \times 10^{-20}$ & $\mathrm{~m}^{2}$ \\
$a_{2}$ & Material gain constant & $7.4 \times 10^{18}$ & $\mathrm{~m}^{-3}$ \\
$a_{3}$ & Material gain constant & $3.0 \times 10^{-32}$ & $\mathrm{~m}^{4}$ \\
$a_{4}$ & Material gain constant & $3.155 \times 10^{25}$ & $\mathrm{~m}^{-4}$ \\
$N_{0}$ & Carrier density at transparency & $1.1 \times 10^{24}$ & $\mathrm{~m}^{-3}$ \\
$\lambda_{0}$ & Wavelength at transparency & $1595 \times 10^{-9}$ & $\mathrm{~m}$ \\
$v_{g}$ & Group velocity & $7.5 \times 10^{7}$ & $\mathrm{~m} / \mathrm{s}$ \\
$d n / d N$ & Differential refractive index & $-1.2 \times 10^{-26}$ & $\mathrm{~m}^{3}$ \\
$\alpha_{c}$ & Loss in claddings & $20 \times 10^{2}$ & $\mathrm{~m}^{-1}$ \\
$\alpha_{s c a t}$ & Scattering loss & $10 \times 10^{2}$ & $\mathrm{~m}^{-1}$ \\
$\Gamma$ & Confinement factor & 0.35 & -- \\
$I$ & Injection current & variable & $\mathrm{A}$ \\
\hline \hline
\end{tabular}

TABLE II

NONLINEAR (FWM) SOA PARAMETERS

\begin{tabular}{cccc}
\hline \hline Symbol & Description & Value & Unit \\
\hline \hline$P_{\text {sat, } C H}$ & CH induced power saturation & 0.69 & $\mathrm{~W}^{-1}$ \\
$P_{s a t, S H B}$ & SHB induced power saturation & 25.5 & $\mathrm{~W}^{-1}$ \\
$\alpha_{C H}$ & Linewidth enhancement factor by $\mathrm{CH}$ & 3.2 & -- \\
$\alpha_{S H B}$ & Linewidth enhancement factor by SHB & -3.6 & -- \\
$\tau_{C H}$ & Carrier heating lifetime & $650 \times 10^{-15}$ & $\mathrm{~s}$ \\
$\tau_{S H B}$ & Spectral hole burning lifetime & $50 \times 10^{-15}$ & $\mathrm{~s}$ \\
\hline \hline
\end{tabular}

$$
\begin{aligned}
\frac{d A_{s 2}}{d z}= & \frac{1}{2}\left[\left\{\Gamma g_{m s_{2}}\left(1-i \alpha_{\mathrm{CM}}\right)-\alpha_{0}\right\}\right. \\
& \left.-\Gamma g_{m s_{2}}\left(\eta_{s 2 p 2}\left|A_{p 2}\right|^{2}+\eta_{s 2 c 2}\left|A_{c 2}\right|^{2}\right)\right] A_{s 2} \\
& -\Gamma \frac{g_{m s_{2}}}{2}\left[\eta_{p 2 c 2} A_{p 2}^{2} A_{c 2}^{*}+\eta_{s 1 p 1} A_{p 2} A_{s 1} A_{p 1}^{*}\right. \\
& \left.+\eta_{p 1 s} A_{p 2} A_{p 1} A_{s}^{*}+\eta_{s 1 s} A_{c 2} A_{s 1} A_{s}^{*}\right] \\
\frac{d A_{p 2}}{d z}= & \frac{1}{2}\left[\left\{\Gamma g_{m p_{2}}\left(1-i \alpha_{\mathrm{CM}}\right)-\alpha_{0}\right\}\right. \\
& \left.-\Gamma g_{m p_{2}}\left(\eta_{p 2 s 2}\left|A_{s 2}\right|^{2}+\eta_{p 2 c 2}\left|A_{c 2}\right|^{2}\right)\right] A_{p 2} \\
& -\Gamma \frac{g_{m p_{2}}}{2}\left[\left(\eta_{s 2 p 2}+\eta_{c 2 p 2}\right) A_{s 2} A_{c 2} A_{p 2}^{*}\right. \\
& +\eta_{s 1 p 1} A_{c 2} A_{s 1} A_{p 1}^{*}+\eta_{p 1 s 1} A_{s 2} A_{p 1} A_{s 1}^{*} \\
& \left.+\eta_{p 1 s} A_{c 2} A_{p 1} A_{s}^{*}+\eta_{s p 1} A_{s 2} A_{s} A_{p 1}^{*}\right] \\
\frac{d A_{c 2}}{d z}= & \frac{1}{2}\left[\left\{\Gamma g_{m c_{2}}\left(1-i \alpha_{\mathrm{CM}}\right)-\alpha_{0}\right\}\right. \\
& \left.-\Gamma g_{m c_{2}}\left(\eta_{c 2 s 2}\left|A_{s 2}\right|^{2}+\eta_{c 2 p 2}\left|A_{p 2}\right|^{2}\right)\right] A_{c 2} \\
& -\Gamma \frac{g_{m c_{2}}}{2}\left[\eta_{p 2 s 2} A_{p 2}^{2} A_{s 2}^{*}+\eta_{p 1 s 1} A_{p 2} A_{p 1} A_{s 1}^{*}\right. \\
& \left.+\eta_{s p 1} A_{p 2} A_{s} A_{p 1}^{*}+\eta_{s s 1} A_{s 2} A_{s} A_{s 1}^{*}\right]
\end{aligned}
$$




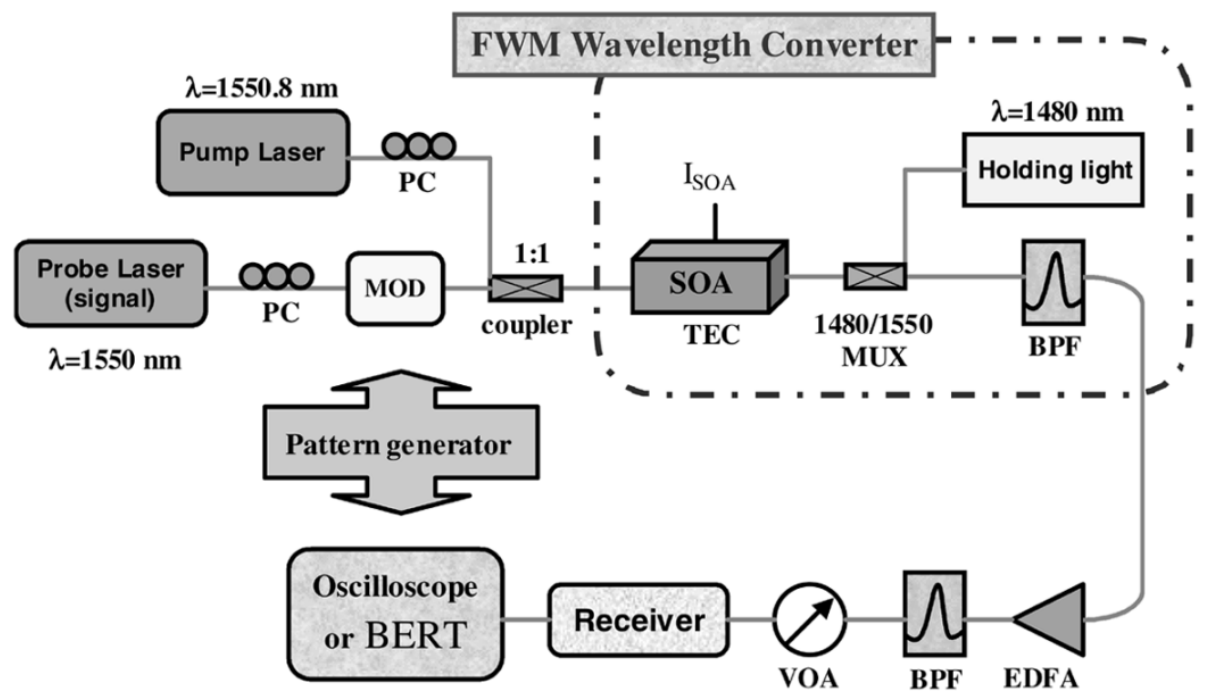

Fig. 3. Experimental setup for measuring dynamic responses of wavelength conversion for the SP scheme.

where $\alpha_{\mathrm{CM}}$ is the linewidth enhancement factor of an SOA induced by carrier density modulation. We assume $\Delta k=2 k_{2}-$ $k_{1}-k_{3} \approx 0$ is satisfied due to the collinear geometry and low dispersion of the SOA [15]. For clarity, the subscript $i$ for the section number has been omitted in (8)-(13).

Higher order wave mixing processes between the newly generated waves and the input waves are neglected by assuming that the input pump power is much stronger than the input signal power. The coefficients $\eta_{j k}, j, k=p, s, c$ are given by [14]

$$
\begin{aligned}
& \eta_{j k}(z)=\frac{\left(\frac{1-i \alpha_{\mathrm{CM}}}{P_{\mathrm{sat}}}\right)}{1+\frac{P(z)}{P_{\mathrm{sat}}}-i\left(\omega_{j}-\omega_{k}\right) \tau_{e}} \\
&+\sum_{m=\mathrm{CH}, \mathrm{SHB}} \frac{\left(\frac{1-i \alpha_{m}}{P_{s, m}}\right)}{1-i\left(\omega_{j}-\omega_{k}\right) \tau_{m}}
\end{aligned}
$$

where $P(z)=\sum_{\omega=p, s, c, a}\left|A_{\omega}(z)\right|^{2}$ is the total optical power inside the amplifier. The saturation power for CM is defined by $P_{\text {sat }}=E_{p} A_{\text {cross }} / \Gamma a_{1} \tau_{e}$, where effective carrier lifetime $\tau_{e}$ is expressed as

$$
\frac{1}{\tau_{e}}=\frac{1}{\tau_{s}}+v_{g} \cdot \sum_{\omega} \frac{g_{m \omega, i} \cdot \Delta L+1}{g_{m \omega, i} \cdot \Delta L} \cdot \frac{d g_{m \omega, i}}{d N} \cdot S_{\omega, i}
$$

The relaxation lifetimes are given by $\tau_{e}$ for carrier modulation as well as $\tau_{\mathrm{CH}}$ and $\tau_{\mathrm{SHB}}$ for carrier heating $(\mathrm{CH})$ and spectral hole burning (SHB), respectively. The saturation power and linewidth enhancement factor are given for each processes. The term $P(z) / P_{\text {sat }}$ appears only in the denominator of the carrier modulation contribution due to the extremely high saturation powers for $\mathrm{CH}$ and SHB processes.

We have taken into account the effect of the holding light $\left(P_{a}\right)$ by using (1). The presence of a holding light changes the carrier density and, thus, the gain coefficient. The injection of a holding light increases $P_{\text {sat }}$, as indicated in (15), which in turn raises the conversion efficiency. The pronounced contribution from the holding light is to the gain coefficient $g_{m \omega},(\omega=s, p$, and $c)$. Under a saturation condition, the optical power depletes carrier density and then decreases the gain. The holding beam can be absorbed and then supplies carriers as gain saturation occurs. For example, if the SOA is biased at the current corresponding to the transparent current of the holding light, the beam generates carriers once the carrier is depleted by the pump and/or signal beams. Holding of the gain under large pump and signal powers result in a significant improvement on the FWM efficiency.

Assuming that the amplitudes of the $\mathrm{P} 2, \mathrm{~S} 2$, and $\mathrm{C} 2$ are zero, it is straightforward to show that (8)-(13) reduce to three basic coupled equations that can describe the single-pump FWM configuration [7]. We have ignored the FWM interaction from the holding light because its wavelength is far away from the active signal zone in which the other beams reside.

Table I lists the simulation and device parameters used in our simulation. A buried-heterostructure (BH) SOA operating in the $1.55-\mu \mathrm{m}$ range is considered for our simulation. The nonlinear SOA parameters are summarized in Table II.

\section{Time-Domain-Modeling Scheme}

Solving the coupled equations in this structure involves obtaining the various traveling-wave amplitudes for each section, where it is assumed that the various material and structural parameters are assumed to be uniform throughout the section. A restriction on the subsections is that they must all have the same length $\Delta z=v_{g} \Delta t$, where $v_{g}$ is the group velocity which effectively sets the time increment for the model. Associated with each section $i$ is a carrier density $N_{i}$ from which the gain $g_{i}$ and the refractive index $n_{i}$ are determined. Incrementing the model in time involves first updating the wave amplitudes at the section boundaries. The subsection carrier densities $N_{i}$ are then updated using Euler's solution of the time-dependent rate equation, as follows:

$$
\begin{aligned}
& N_{i}(t+\Delta t)=N_{i}(t)+\Delta t \\
& \quad \cdot\left[\frac{J}{e d}-R\left(N_{i}\right)-\sum_{\omega} \Gamma v_{g} g_{m \omega, i} S_{\omega, i}-R_{\mathrm{ASE}}\left(N_{i}\right)\right] .
\end{aligned}
$$

As a starting point for the simulation, the carrier densities $N_{i}$ in each section is obtained using the carrier density rate (1) by neglecting the stimulated and spontaneous emission. Solving $N_{i}$ 
allows us to calculate the FWM coupling coefficient $\eta_{j k, i}$ as well as the gain coefficient $g_{m \omega, i}$ for every section. The traveling-wave amplitudes $A_{\omega, i+1}$ for the $(i+1)$ th section can be calculated by solving the coupled (8)-(13), which comprise the FWM interactions. The evolution of the holding light is calculated simply by propagating it from the output end to the input end. The backward and forward ASEs are calculated by dividing the major spectral content into 32 uniform slices. The average photon densities $S_{\omega, i}$ are then calculated from $A_{\omega, i}$, and $A_{\omega, i+1}$, using (2), and the ASE recombination rate is calculated using (6). Finally, the carrier densities $N_{i}$ are updated by iteration using (1).

The first step is to initialize the model, and this is followed by a straightforward iterative loop with the traveling-wave amplitudes and carrier densities being updated at each time increment. The updating procedure involves first resolving the coupled equations, and this is followed by updating the traveling-wave amplitudes $A_{\omega}$ at the subsection boundaries. From the convergent wave amplitudes, the output power is calculated. Finally, the subsection carrier densities $N_{i}$ are updated. The time $t$ is then incremented by $\Delta t$, and the whole cycle is repeated. We set the time interval $d t$ and one section length $d z$ as $0.5 \mathrm{ps}$ and $37 \mu \mathrm{m}$ to assure enough accuracy in the time step up to $10 \mathrm{~Gb} / \mathrm{s}$.

\section{EXPERIMENTAL SETUP FOR TRANSMisSiOn TEST}

The setup for measuring the SP scheme with a holding light is shown in Fig. 3. The pump and probe beams produced from two commercial tunable lasers with built-in isolators were combined using a 50/50 coupler and lunched into an SOA. A 1.48/1.55- $\mu \mathrm{m}$ multiplexer was used at the output end to allow the injection of a holding light and the output of the converted signal. The multiplexer provides low insertion loss to the 1480-nm holding light. The SOA is a fiber pigtailed unit from Alcatel Corporation with 23-dB fiber-to-fiber gain. The SOA is polarization independent, but both the pump and probe polarizations must be controlled to obtain the best wavelength conversion efficiency. The measurement for the OPP scheme can be performed similarly by adding the second pump beam.

A Mach-Zehnder external modulator is used for encoding the pseudorandom-binary-sequence (PRBS) data onto the probe light. After optical filtering to reject the residual pump and probe signals, the converted signals are amplified by an erbium-doped fiber amplifier (EDFA). The amplified signals pass through another bandpass filter of 0.26-nm bandwidth to suppress the ASE of the EDFA. The converter output is then detected with a highspeed optical receiver.

In the experiment, a holding power of $23.5 \mathrm{dBm}$ is used. The probe wavelength is $1550 \mathrm{~nm}$. Wavelength conversion was evaluated at two different bias currents, one at transparency $(105 \mathrm{~mA})$ for the holding light and the other at high injection (200 mA).

\section{DyNAMIC ReSPONSES FOR THE SINGLE-PumP SCHEME}

\section{A. Simulation Results}

In the simulation, super-Gaussian pulses $(m=3)$ with a $2^{7}-1$ PRBS data pattern are used. The input signal power is $0.1 \mathrm{~mW}$ and the input data rate is $10 \mathrm{~Gb} / \mathrm{s}$. The holding light

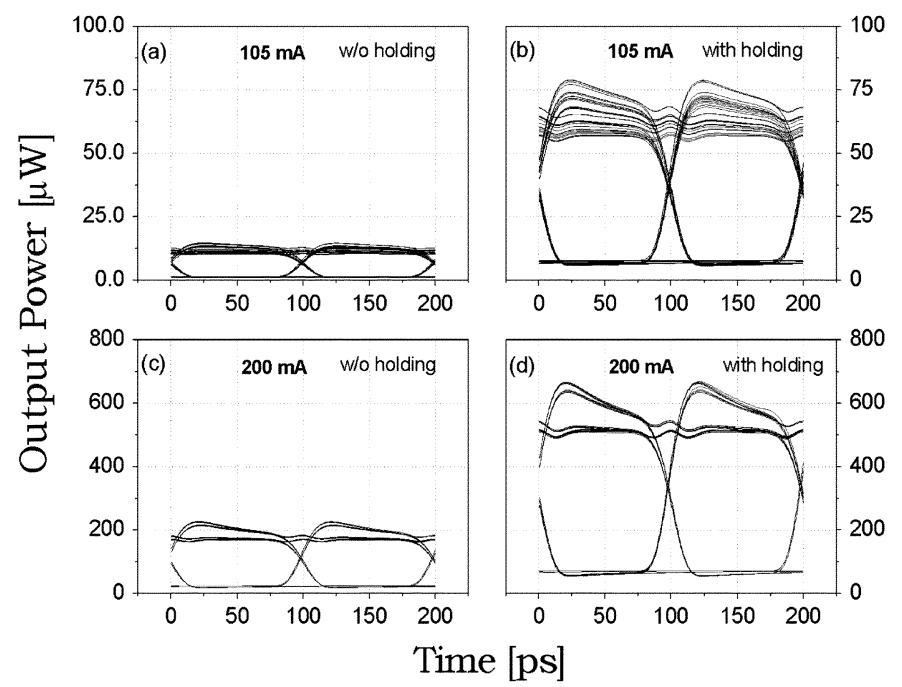

Fig. 4. Eye diagram for single-pump FWM in LHSOAs. The SOA is biased at $I=105 \mathrm{~mA}$ (a) without and (b) with the holding light, as well as at $I=$ $200 \mathrm{~mA}$ (c) without and (d) with a 1480-nm holding light, respectively.

power is $23 \mathrm{dBm}$. The wavelengths for the pump and signal are 1549 and $1550 \mathrm{~nm}$, respectively. The input pump power is $-3 \mathrm{dBm}$. Fig. 4 shows the corresponding eye diagrams of the conjugated signals. Injecting a holding light increases the amplitude of converted signal. The holding light can provide larger improvement when the SOA is biased at the corresponding transparency $(105 \mathrm{~mA})$, in comparison to the case of biasing at $200 \mathrm{~mA}$ [6]. For both bias conditions, the output waveform is not affected by the injection of a holding light.

Fig. 5 shows the frequency chirp for the FWM in an SOA with and without a holding light. Frequency chirp arises from the refractive index modulation induced by variations of the carrier density. The peak chirp of the conjugate signal with a holding light is only slightly larger than that for one without the holding light, while the efficiency is significantly increased. This indicates that the holding light stabilizes the carrier density in response to an input modulated light. The conjugate signal have negative chirp, which may be used for compensating the fiber dispersion.

\section{B. Experimental Data}

Fig. 6 compares the eye diagrams for the conjugated signals without and with a holding light. The SOA is biased at transparency $\left(I_{\mathrm{SOA}}=105 \mathrm{~mA}\right)$. It can be clearly seen that the injected holding light increases the converted signal amplitude by about $5 \mathrm{~dB}$. We attribute this to the enhanced conversion efficiency ( $\sim 5.2 \mathrm{~dB})[6]$. The eye diagrams of the FWM conjugated signal in the case of 200-mA bias are displayed in Fig. 7. The rise of $\sim 1.4 \mathrm{~dB}$ in amplitude for the FWM conjugated signal is attained by injecting a holding light.

From the comparisons between the results shown in Figs. 6 and 7, a clearer eye opening can be found when biasing the SOA at high current density, which has a larger SBR. However, at a higher SOA bias, the enhancement by the holding light is getting smaller. This is due to the fact that the holding light increases the saturation power but decreases the unsaturated gain. Signal distortion and less eye opening occur for converting $10-\mathrm{Gb} / \mathrm{s}$ sig- 


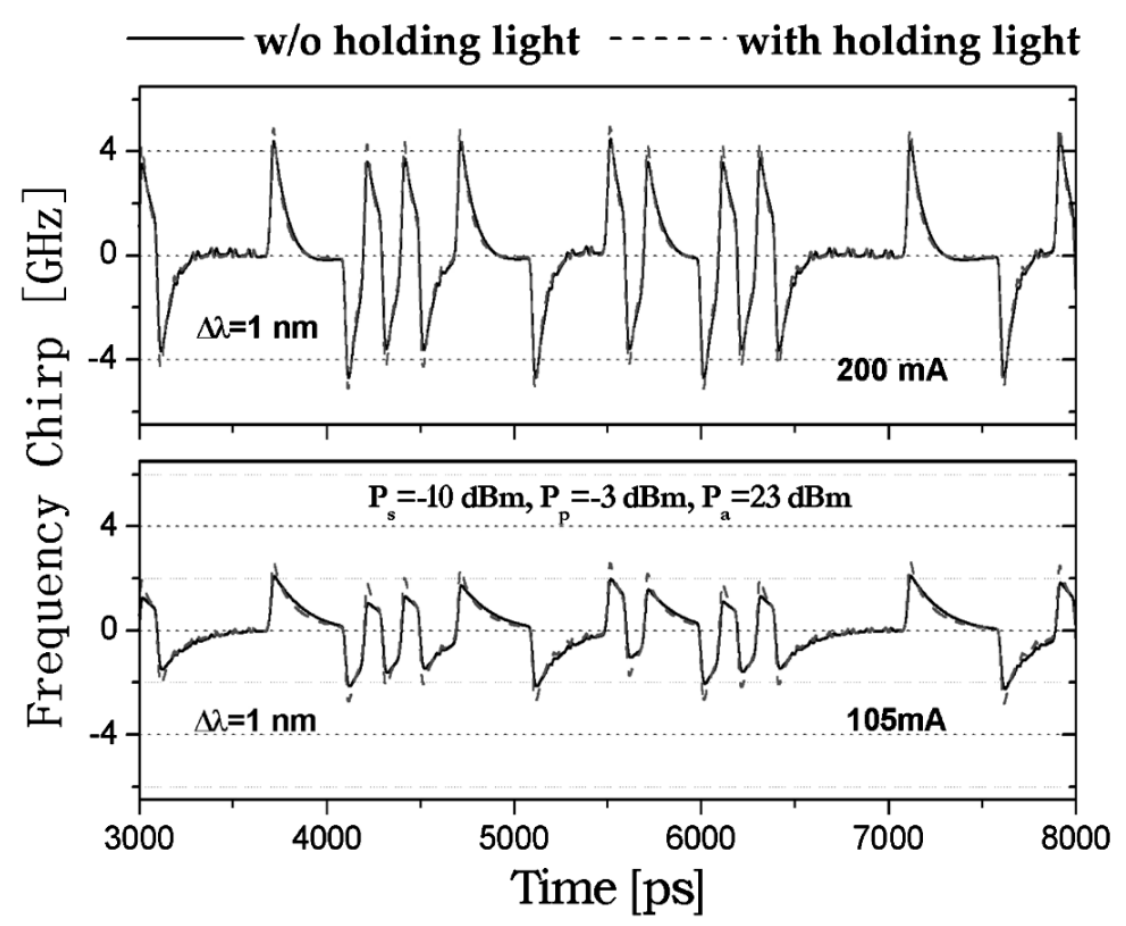

Fig. 5. Frequency chirps of the output conjugate signals for the single-pump FWM in LHSOAs.

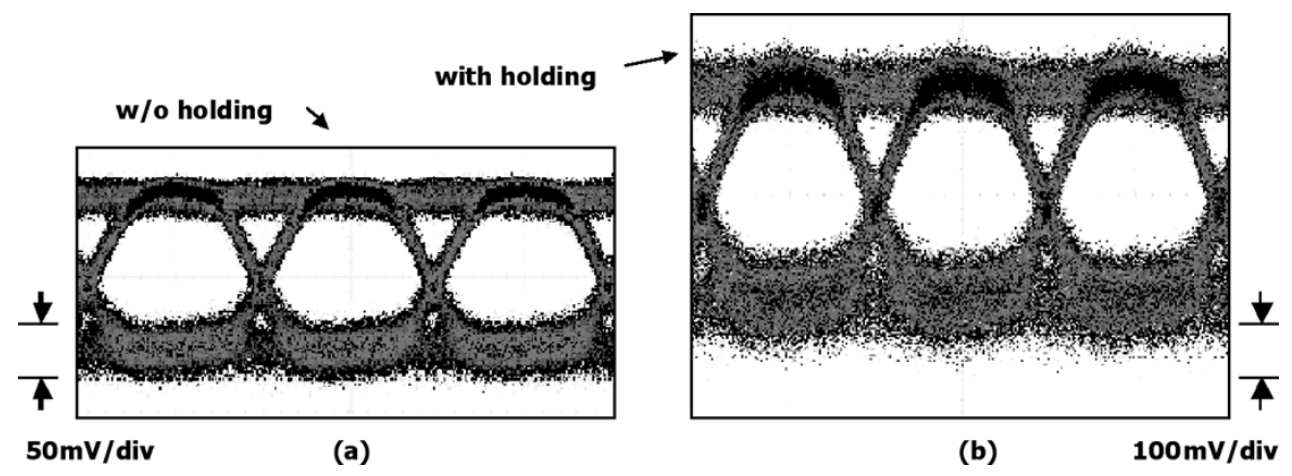

Fig. 6. Eye diagrams of the converted signals at $2.5 \mathrm{~Gb} / \mathrm{s}$ : (a) without and (b) with a holding light. $I_{\mathrm{SOA}}=105 \mathrm{~mA}, P_{\mathrm{p}}=-2 \mathrm{dBm}, P_{\mathrm{b}}=-7 \mathrm{dBm}, P_{\mathrm{a}}=23.5$ $\mathrm{dBm}$ and $\lambda_{\mathrm{a}}=1480 \mathrm{~nm}$. The waveforms are inverted.

nals. The measured waveforms resemble the simulated results, as shown in Fig. 4. Therefore, the signal distortion is mostly resulted from the residual pattern effect and the limited response time of the SOA. Eye-opening degradation is partly due to the incomplete suppression of the ASE and the absence of a $10-\mathrm{GHz}$ electrical filter after the receiver. The experimental results indicate that the use of a holding light does not affect the shape of the conjugated signal waveform.

Both the calculated and measured eye diagrams indicate that the most pronounced effect of using a holding light is the increase of conversion efficiency. Though a holding light also enhances the SBR and effective carrier lifetime, the effects cannot clearly be observed from the eye diagram without detailed analysis on the signal waveforms.

In addition to the improvement on conversion efficiency, the holding light can increase the SBR by about $3 \mathrm{~dB}$ and $1.5 \mathrm{~dB}$ at the bias levels of 105 and $200 \mathrm{~mA}$, respectively [6]. The holding light provides a greater enhancement on the signal amplitude than on the ASE. Since both the efficiency and SBR are improved, using a holding light can reduce the power penalty caused by the wavelength conversion process.

To characterize the power penalty associated with the wavelength conversion, we conducted BER measurements with $2^{31}-1$ PRBS signals. The results are shown in Fig. 8 for down conversion (to a shorter wavelength). Without using an holding light, the power penalty at $\mathrm{BER}=10^{-10}$ for $2.5 \mathrm{~Gb} / \mathrm{s}$ is about $2.1 \mathrm{~dB}$ [Fig. 8(a)], which is mainly incurred from the ASE of the SOA. The power penalty increases to about $4.6 \mathrm{~dB}$ for $10-\mathrm{Gb} / \mathrm{s}$ signals, which indicates that the signal is also impaired by the pattern effect for high data rates. With the injection of a holding light to promote the SBR of the SOA, the power penalty decreases by the amount of $0.9 \mathrm{~dB}$ and $2.2 \mathrm{~dB}$ for $2.5-\mathrm{Gb} / \mathrm{s}$ and $10-\mathrm{Gb} / \mathrm{s}$ data rates, respectively. Owing to the lack of $10-\mathrm{GHz}$ electrical filters, the BER tester (BERT) receives a wider spectral range of noise than the signal bandwidth. This contributes to the larger power penalty at $10 \mathrm{~Gb} / \mathrm{s}$ than at $2.5 \mathrm{~Gb} / \mathrm{s}$. The analysis of eye diagrams also illustrates such an effect. 


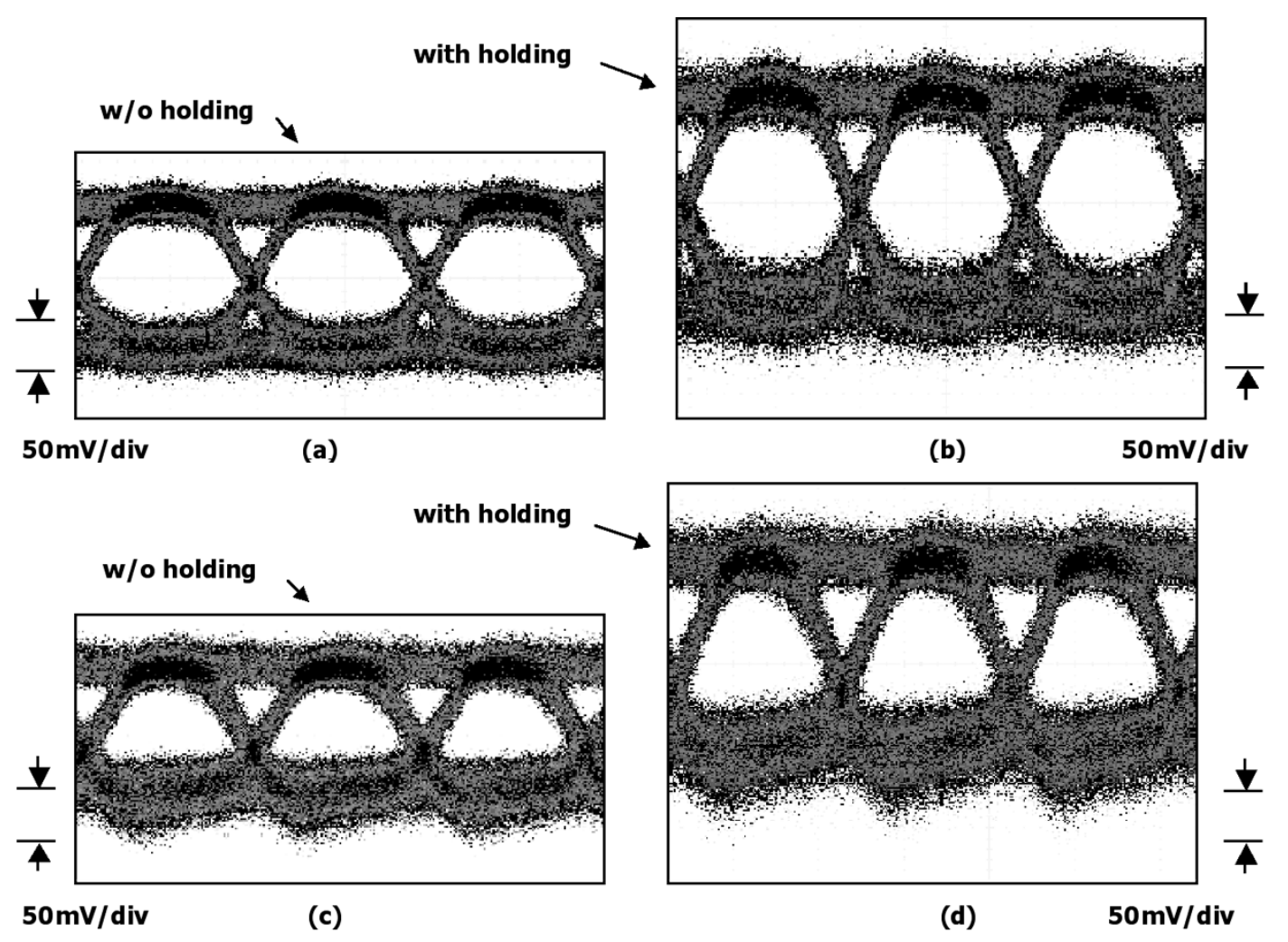

Fig. 7. Eye diagrams of the wavelength converted signal at $2.5 \mathrm{~Gb} / \mathrm{s}$ PRBS (a) without and (b) with a holding light as well as at $10 \mathrm{~Gb} / \mathrm{s}$ PRBS (c) without and (d) with assist light. $I_{\mathrm{SOA}}=200 \mathrm{~mA}, P_{\mathrm{p}}=-2 \mathrm{dBm}, P_{\mathrm{b}}=-7 \mathrm{dBm}, P_{\mathrm{a}}=23.5 \mathrm{dBm}$, and $\lambda_{\mathrm{a}}=1480 \mathrm{~nm}$.

Fig. 9 shows the BER characteristics at high SOA bias condition $\left(I_{\mathrm{SOA}}=200 \mathrm{~mA}\right)$. Without a holding light, the power penalties after wavelength conversion for both modulation rates are almost equal, $1.2 \mathrm{~dB}$, which is smaller than those in Fig. 8. The better SBR at a higher bias level is the major cause. Moreover, biasing the SOA at higher current density accelerates the gain recovery, leading to a reduced patterning effect and ISI. At such a condition, the extent of the power penalty to be compensated by using a holding light is, thus, in shrinkage $(\sim 0.2 \mathrm{~dB})$. Noting that the holding light not only reduces the power penalty but also improves the conversion efficiency.

\section{OPP FWM IN AN LHSOA}

We apply our simulation model to the OPP scheme in order for investigating its conversion efficiency and SBR. We then show that the models in Section I can describe the static and dynamic responses of the OPP FWM with a holding light.

We first consider the case without a holding beam. Due to the interactions among the three input waves, a number of FWM products are generated. The S2 is the dual-pump phase conjugated product [16] of the input signal. Theoretical analysis of the OPP conversion scheme shows that the converted signal S2 is mainly generated as a result of the scattering of $\mathrm{P} 2$ by the beating between the signal and $\mathrm{P} 1$. The magnitude of this process is dependent on the wavelength spacing between the signal and P1 $\Delta \lambda$, which is fixed. Thus, the wavelength conversion efficiency is expected to be independent of $\mathrm{P} 2$ wavelength. The mixing process between the two pumps has negligible contribution to
S2, because the two pumps are orthogonally polarized and relatively far apart.

We calculated the FWM conversion efficiency and SBR of the converted signal (S2) by sweeping P2 to cover a wavelength band from 1515 to $1595 \mathrm{~nm}$. Fig. 10 shows the calculated conversion efficiency and SBR versus the wavelength shift for the OPP schemes. The optical power for S, P1, and P2 are $-10,-7$, and $-3 \mathrm{dBm}$, respectively. We fix $\Delta \lambda$ to be $1 \mathrm{~nm}$ with $\lambda_{\mathrm{P} 1}$ and $\lambda_{\mathrm{S}}$ are 1554 and $1555 \mathrm{~nm}$, respectively. At a bias current of $200 \mathrm{~mA}$, high conversion efficiency and SBR $(-5 \mathrm{~dB}$ and $25 \mathrm{~dB}$ ) can be obtained, as shown in Fig. 10. The conversion efficiency for the OPP scheme is almost constant over a wide conversion range. Injecting a holding light further modifies the gain spectrum so that the conversion efficiency and SBR could still be enhanced by around 5 and $2 \mathrm{~dB}$, respectively. The conversion efficiency is better than $-10 \mathrm{~dB}$ for $>80 \mathrm{~nm}$ of wavelength range. The holding light also improves the flatness of the conversion efficiency, so the efficiency is almost constant over $60 \mathrm{~nm}$ of conversion range. A nearly constant $(<3 \mathrm{~dB})$ SBR over a $80-\mathrm{nm}$ range is also achieved. This is again benefited from the increased saturation power [6].

We note that the conversion efficiency drops at wavelengths far from the gain peak of the SOA. This effect is due to the reduction in the gain of the SOA. A wider conversion bandwidth may be attained by using an SOA with a wider material gain spectrum. The conversion efficiency can further be enhanced with an increase in the pump power and a decrease in $\Delta \lambda$. There is no corresponding drop in SBR at larger wavelength shifts, since both the converted signal and the noise are proportional to the gain. 


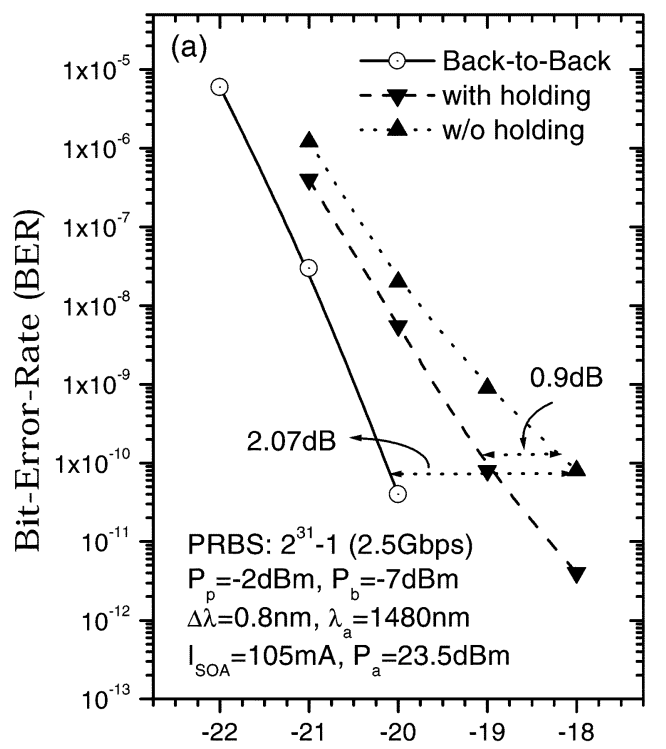

Average receiver power $[\mathrm{dBm}]$

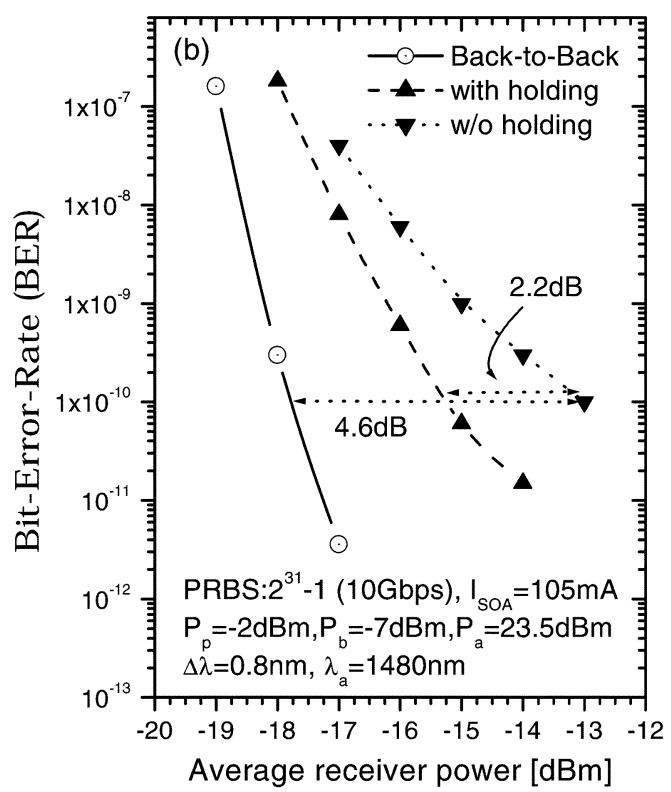

Fig. 8. BER characteristics of FWM converted signal from an SOA biased at transparency $\left(I_{\mathrm{SOA}}=105 \mathrm{~mA}\right)$ at: (a) 2.5 and (b) $10 \mathrm{~Gb} / \mathrm{s}$.

We also examine the dynamic responses of the OPP FWM wavelength conversion using our simulation model. The signal power is $0.2 \mathrm{~mW}$. The calculated conjugated pulse waveforms and their corresponding eye diagrams for $30-\mathrm{nm}$ wavelength conversion are displayed in Fig. 11 for the cases with and without using the holding light. In contrast to the single-pump FWM, the OPP scheme give a clearer eye with reduced pattern effect on high level and suppressed ASE noise on low level. Injecting a holding light further enlarges the eye opening because it improves not only the conversion efficiency but also the SBR. The simulation results are again very consistent with the experimental results that have been addressed elsewhere [17].

To address the effect of the holding light, we compare the performance improvement at different bias currents by using a holding light in Fig. 12. The enhancement decreases with the increasing bias due to the fact that the holding light increases the
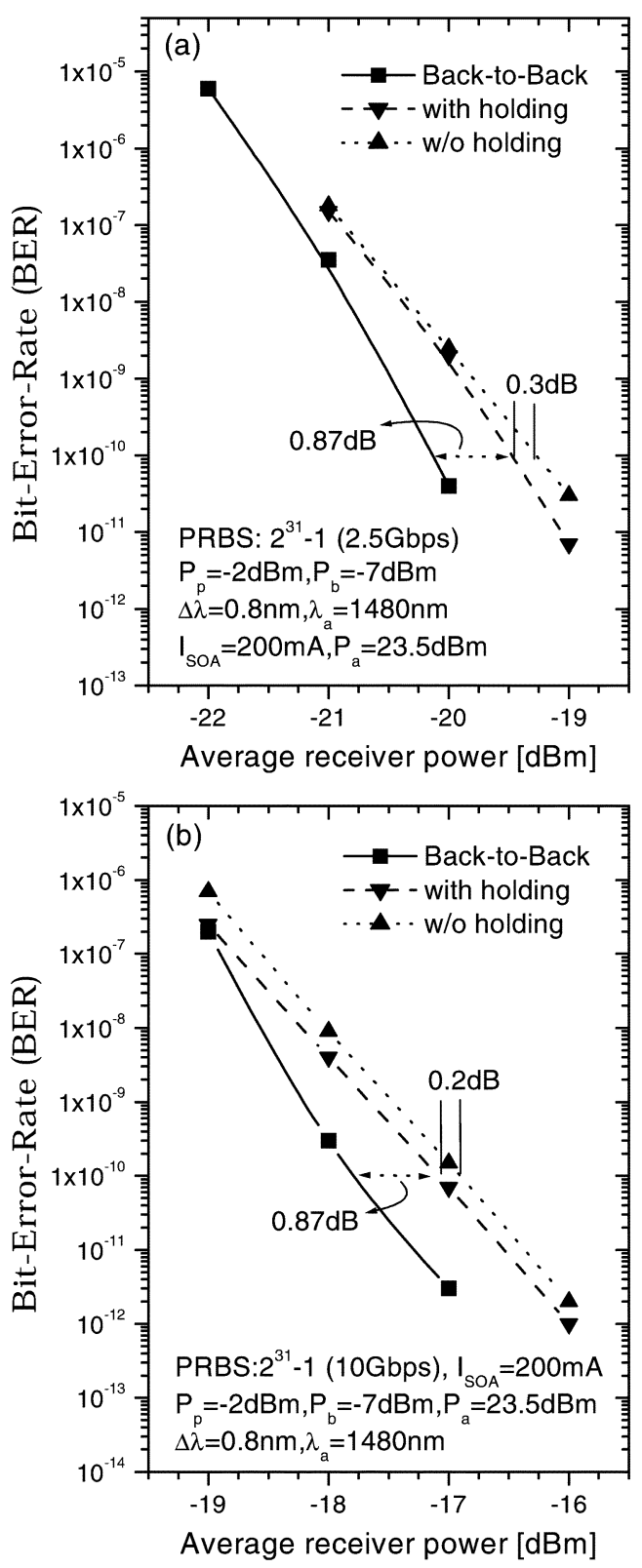

Fig. 9. BER characteristics of FWM converted signal from an SOA biased at gain region $\left(I_{\mathrm{SOA}}=200 \mathrm{~mA}\right)$ at: (a) 2.5 and (b) $10 \mathrm{~Gb} / \mathrm{s}$.

saturation power but decreases the available gain at high bias. The strategy of using an SOA as an FWM wavelength converter is to operate it at the rated current to achieve the highest conversion efficiency and SBR. However, the SOA gain saturates at high bias, so the efficiency improves only slightly by simply increasing the current. Operating an SOA at rated current may shorten its lifetime. Fig. 12 indicates that biasing the SOA at $135 \mathrm{~mA}$ with a holding light can achieve the same conversion efficiency and SBR as one biased at $200 \mathrm{~mA}$ without the holding light.

The results for biasing the SOA at $105 \mathrm{~mA}$, transparent current for the 1480-nm holding light, indicate that the holding light can provide considerable improvement on the wavelength conversion. At such a condition, the holding light increases the SOA saturation intensity without sacrificing the gain. To provide the 


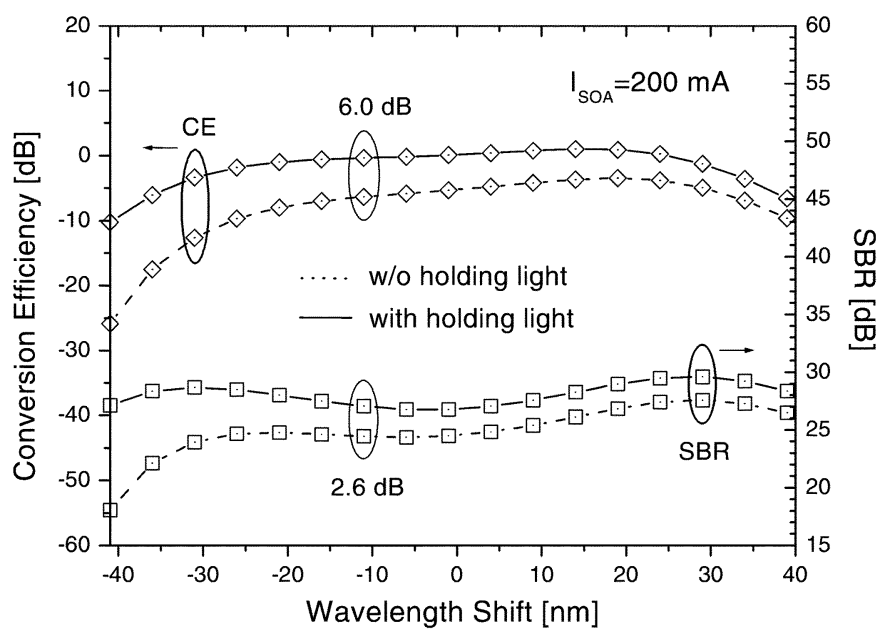

Fig. 10. Effects of the holding light on conversion efficiency (CE) and SBR. The SOA is biased at $200 \mathrm{~mA}$.

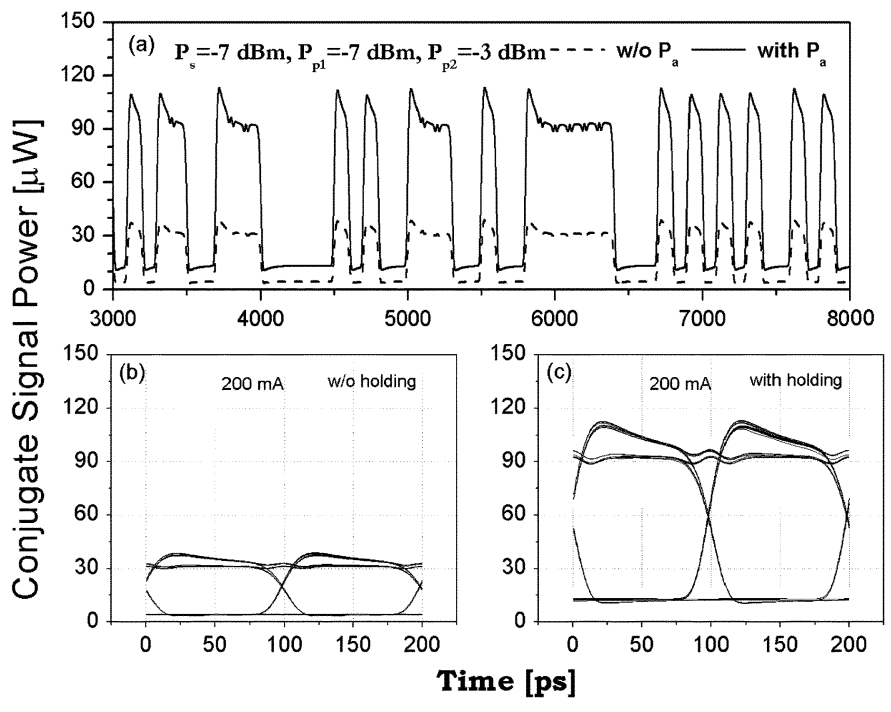

Fig. 11. (a) Output conjugated pulse patterns and eye diagram for 30-nm OPP FWM wavelength conversion in LHSOAs (b) without and (c) with a 1480-nm holding light. The wavelengths for the pump 1, pump 2, and signal are 1554, 1526 , and $1555 \mathrm{~nm}$, respectively. The SOA is biased at $I=200 \mathrm{~mA}$ and the holding light power is $23 \mathrm{dBm}$.

same improvement at higher biases, where conversion efficiency is better, a shorter wavelength holding light must be used.

\section{CONCLUSION}

We have experimentally and theoretically investigated the FWM-based wavelength converter using an SOA in combination with a holding light. Two FWM schemes are studied here. Under a single-pump FWM scheme, through the injection of a shorter wavelength holding light, we can provide performance improvements at higher gain, which is needed for high-efficiency and low-noise FWM wavelength conversion. Dynamic measurement reveals that using a holding light can reduce the power penalty by $2.2 \mathrm{~dB}$. Comparisons between the experimental and simulated results indicate that the converted SBR and the pattern effect are the main factors affecting the BER performance. On the other hand, installing the OPP FWM scheme in LHSOAs will give higher conversion efficiency

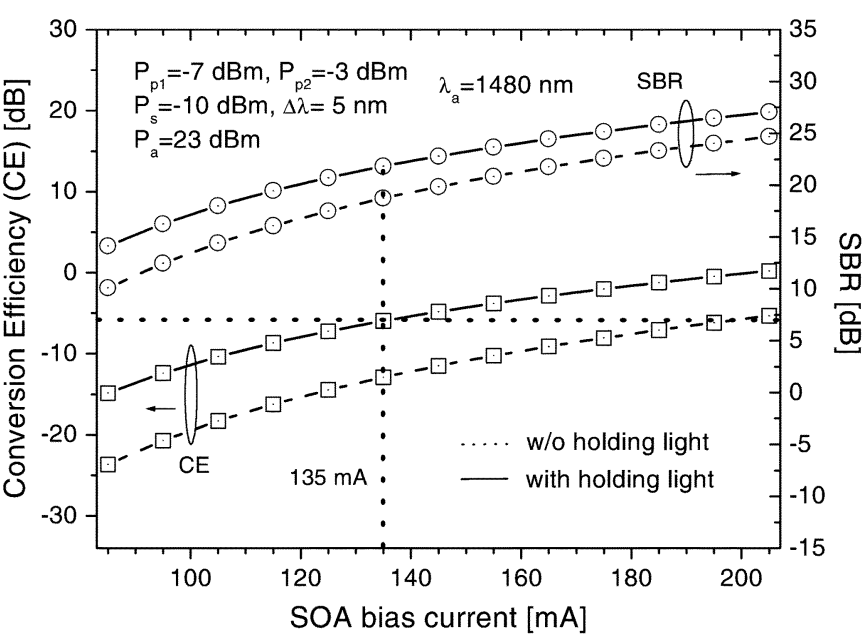

Fig. 12. Effects of the holding light on conversion efficiency (CE) and SBR versus SOA bias currents.

and SBR over a wide range of wavelength shifts. By injecting a holding light, we can, thus, raise the saturation power, enhance the conversion efficiency, flatten the efficiency against wavelength, improve the dynamic characteristics, and extend the conversion bandwidth of FWM wavelength converters. These merits will make the wavelength conversion feasible for practical applications.

It is shown that the theoretical models developed in this paper can describe the dynamic and static responses very well. The model can be used for further optimizing the wavelength converters using either the SP or OPP scheme. The optimization parameters can include the SOA length, the wavelength of the holding light, and the configuration of the pumps and holding light.

\section{REFERENCES}

[1] D. F. Geraghty, R. B. Lee, M. Verdiell, M. Ziari, A. Mathur, and K. J. Vahala, "Wavelength conversion for WDM communication systems using four-wave mixing in semiconductor optical amplifiers," IEEE $J$. Select. Topics Quantum Electron., vol. 3, pp. 1146-1155, Oct. 1997.

[2] A. D'Ottavi, A. Mecozzi, S. Scotti, F. C. Romeo, F. Martelli, and P. Spano, "Four-wave mixing efficiency in traveling wave semiconductor optical amplifiers at high saturation," Appl. Phys. Lett., vol. 67, pp. 2753-2755, 1995.

[3] T. J. Morgan, P. R. Lacey, and R. S. Tucker, "Widely tunable four-wave mixing in semiconductor optical amplifiers with constant conversion efficiency," IEEE Photon. Technol. Lett., vol. 10, pp. 1401-1403, Oct. 1998.

[4] I. Tomkos, I. Zacharopoulos, D. Syvridis, Th. Sphicopoulos, C. Caroubalos, and E. Roditi, "Performance of a reconfigurable wavelength converter based on dual-pump-wave mixing in a semiconductor optcal amplifier," IEEE Photon. Technol. Lett., vol. 10, pp. 1404-1406, Oct. 1998.

[5] A. D'Ottavi, F. Girardin, L. Graziani, F. Martelli, P. Spano, A. Mecozzi, S. Scotti, R. Dall'Ara, J. Eckner, and G. Guekos, "Four-wave mixing in semiconductor optical amplifiers: A practical tool for wavelength conversion," IEEE J. Select. Topics Quantum Electron., vol. 3, pp. 522-528, Apr. 1997.

[6] S. L. Lee, P. M. Gong, and C. T. Yang, "Performance enhancement on SOA-based four-wave-mixing wavelength conversion using an assisted beam," IEEE Photon. Technol. Lett., vol. 14, pp. 1713-1715, Dec. 2002

[7] P. M. Gong, J. T. Hsieh, S. L. Lee, and J. Wu, "Theoretical analysis of wavelength conversion based on four-wave-mixing in light-holding SOA's," IEEE J. Quantum Electron., vol. 40, pp. 31-40, Jan. 2004.

[8] A. E. Willner and W. Shieh, "Optimal spectral and power parameters for all optical wavelength shifting: Single stage, fanout, and cascadability," J. Lightwave Technol., vol. 13, pp. 771-781, May 1995. 
[9] Y. Suematsu and A. R. Adams, Handbook of Semiconductor Laser and Photonic Integrated Circuits. London, U.K.: Chapman \& Hall, 1994.

[10] G. P. Agrawal and N. K. Dutta, Semiconductor Lasers. New York: Van Nostrand, 1993.

[11] K. Obermann, I. Koltchanov, K. Petermann, S. Diez, R. Ludwig, and H. G. Weber, "Noise analysis of frequency converters utilizing semiconductor optical amplifiers," IEEE J. Quantum Electron., vol. 33, pp. 81-88, Jan. 1997.

[12] T. Mukai, Y. Yamamoto, and T. Kimura, "S/N and error rate performance in AlGaAs semiconductor laser preamplifier and linear repeater systems," IEEE Trans. Microwave Theory Tech., vol. MTT-30, pp. 1548-1556, Oct. 1982.

[13] M. A. Summerfield and R. S. Tucker, "Frequency-domain model of multiwave mixing in bulk semiconductor optical amplifiers," IEEE J. Select. Topics Quantum Electron., vol. 5, pp. 839-850, May/June 1999.

[14] G. P. Agrawal, "Population pulsations and nondegenerate four-wave mixing in semiconductor lasers and amplifiers," J. Opt. Soc. Amer. B, vol. 5, pp. 147-158, 1988 .

[15] J. Zhou, N. Park, J. W. Dawson, K. J. Vahala, M. A. Newkirk, and B. I. Miller, "Efficiency of broadband four-wave mixing wavelength conversion using semiconductor traveling-wave amplifiers," IEEE Photon. Technol. Lett., vol. 6, pp. 50-52, Jan. 1994.

[16] G. Hunziker, P. Paiella, D. F. Geraghty, K. J. Vahala, and U. Koren, "Polarization-independent wavelength conversion at $2.5 \mathrm{~Gb} / \mathrm{s}$ by dual-pump four-wave mixing in a strained semiconductor optical amplifier," IEEE Photon. Technol. Lett., vol. 8, pp. 1633-1635, Dec. 1996.

[17] D. Z. Hsu, S. L. Lee, P. M. Gong, Y. M. Lin, S. S. W. Lee, and M. C. Yuang, "High-efficiency and wideband SOA-based wavelength converters by using four-wave-mixing with orthogonal pumps and an assist beam," IEEE Photon. Technol. Lett., vol. 16, pp. 1903-1905, Aug. 2004.
Jyh-Tsung Hsieh, photograph and biography not available at the time of publication.

Pei-Miin Gong, photograph and biography not available at the time of publication.

San-Liang Lee (S'92-M'95), photograph and biography not available at the time of publication.

Jingshown Wu (S'73-M'78-SM'99), photograph and biography not available at the time of publication. 